\title{
Autologous Anti-BCMA CAR T-cells IM21
}

National Cancer Institute

\section{Source}

National Cancer Institute. Autologous Anti-BCMA CAR T-cells IM21. NCI Thesaurus.

Code C160704.

A preparation of autologous T-lymphocytes that have been transduced with a lentiviral vector (LV) expressing a chimeric antigen receptor (CAR) targeting the human tumorassociated antigen (TAA) B-cell maturation antigen (BCMA; tumor necrosis factor receptor superfamily member 17; TNFRSF17) and containing, as of yet undisclosed costimulatory signaling domains, with potential antineoplastic activity. Upon administration, the autologous anti-BCMA CAR T-cells IM21 recognize and induce selective toxicity against BCMA-expressing tumor cells. BCMA, a tumor-specific antigen and a receptor for both a proliferation-inducing ligand (APRIL) and B-cell activating factor (BAFF), is a member of the tumor necrosis factor receptor superfamily (TNFRSF) and plays a key role in plasma cell survival. BCMA is found on the surfaces of plasma cells and is overexpressed on malignant plasma cells. 